\title{
Indoor Scanning and Mapping using Mobile Robot and RP Lidar
}

\author{
Marni Azira Markom ${ }^{1}$, Shazmin Aniza Abdul Shukor ${ }^{1}$, Abdul Hamid Adom ${ }^{1}$, \\ Erdy Sulino Mohd Muslim Tan ${ }^{1}$, and Ali Yeon Md Shakaff ${ }^{2}$
}

\begin{abstract}
The environment scanning has been important in a mobile robot studies and applications that may help people to investigate, monitor and study a variety of environments such as an unexplored, hazardous, dynamic, cluttered and others. The main issue regarding this research is the efficiency of the mobile robot system and the accuracy of the front-end sensor. This paper presents a work on the development of a mobile robot for the environment scanning purposes where a laser range finder (LRF) is utilized as its scanning sensor. The development of the mobile robot system will be explained here which consists of the hardware / software, the path following navigation and the integration of the LRF system. Three environments were used as the testbed - a corridor, a research lab and a robotic lab. The scanning results are presented in local map and global map which are plotted by using MATLAB software. It is found out that the plotted maps are similar to the real environment, which can be concluded that the developed mobile robot with the LRF is a success and can be used in real applications.
\end{abstract}

Keywords-indoor scanning, mapping, mobile robot and RP Lidar.

\section{INTRODUCTION}

$\mathrm{N}$ OWADAYS, autonomous mobile robot has been widely used in many sectors, from household tasks, light and heavy industries, to the underwater / undersea and outer space. The use of autonomous mobile robot is very beneficial, organized and time saving, especially in dangerous and difficult situations. In the future, it is expected for the autonomous mobile robot to increase its work fields compared to the presents, in accordance with the growing of high technology, complex computational and wireless operational.

In the late 90's, the robotics research has been started to use a laser range finder (LRF) as its main front-end sensor. The use of LRF is quite reliable and accurate compared to other sensors such as ultrasonic and odometer. By using an LRF, a number of tasks can be accomplished, such as mapping and modeling the environments $[1,2,3,4]$, obstacles detection [5,6], robot's motion estimation [7,8] and position $[9,10]$, target and object tracking $[11,12]$, and robot's selflocalisation [13].

In year 2000's till now, there are two brands of LRF that

${ }^{1}$ School of Mechatronic Engineering, Universiti Malaysia Perlis, 02700 Pauh, Perlis, MALAYSIA

${ }^{2}$ Centre of Excellence for Advance Sensor Technology, Universiti Malaysia Perlis, Taman Muhibah, 02600 Jejawi, Perlis, MALAYSIA always been used for robotic studies, which are Sick and Hokuyo. These two brands provide LRF that can be used for indoor and outdoor applications. For indoor purposes, the most LRF that always been used is within the range of $4 \mathrm{~m}$ to $6 \mathrm{~m}$. While outdoors, it may be increased up to $80 \mathrm{~m}$. The issue with this both brands of LRF is that they are expensive. For Hokuyo laser with $4 \mathrm{~m}$ scanning range, it is costs about MYR 8,000 to MYR 9,000 per unit [14]. The cost will rise for increasing scanning range. For Sick laser, most of them are developed with a far scanning range such as $20 \mathrm{~m}$ and $80 \mathrm{~m}$. These scanning ranges are not suitable for indoor, thus making them commonly being used for outdoor purposes. As the scanning range is quite distant, the prices are also high. In Malaysia, a laser with $20 \mathrm{~m}$ scanning range will cost about MYR 20,000 and over.

Then, in the year 2012, Robopeak, a company from China, developed a simple and cheap LRF, called RP Lidar. RP Lidar is a 360 degree scanner that can scan up to $6 \mathrm{~m}$, making it suitable for indoor study and application. The cost per unit is around MYR 1,700 [14]. This laser is available in the world market since the last couple of years. Due to this, there are not many publications and researches can be found. Fig. 1 shows the three types of LRF that been used for indoor study.

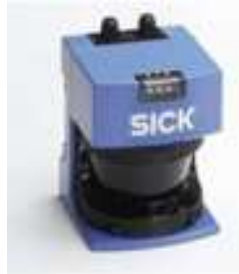

(a)

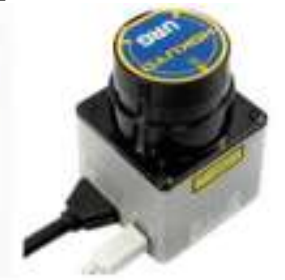

(b)

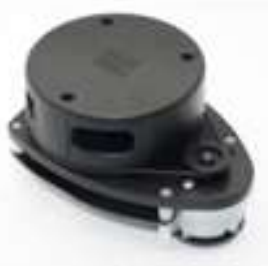

(c)
Fig. 1: (a) Sick LRF, (b) Hokuyo LRF, and (c) RP Lidar

This paper presents on the development work of a mobile robot with an LRF for environment scanning purposes. The first objective of this research is to show the integration process of the mobile robot with the LRF. The second objective is to show the scanning and mapping work. The mobile robot is developed with a wireless system in order to collect the laser data wirelessly. Navigation of the mobile robot, together with the scanning, data collection and the mapping methods will be shown here. The results as well as the performance of the mobile robot and the LRF will be discussed here. 


\section{The Development of The Mobile Robot And The MONIORING SYSTEM}

Nowadays, autonomous mobile robot has been widely used in many sectors, from household tasks, light and heavy industries, to the underwater / undersea and outer space. The use of autonomous mobile robot is very beneficial, organized and time saving, especially in dangerous and difficult situations. In the future, it is expected for the autonomous mobile robot to increase its work fields compared to the presents, in accordance with the growing of high technology, complex computational and wireless operational.

There are two parts that need to be considered during the development of this mobile robot for scanning purposes. The first part would be the development of the mobile robot itself and the second part is the monitoring / control part. The system is designed to be wireless in order to make it easier to navigate and move in the particular environment. The mobile robot will scan and save the environment data surrounding it at the same time, while the monitoring / control part will monitor the mobile robot's movement and scanning results as well as for controlling the system.

The development of the mobile robot is based on a typical

mobile robot. It consists of a few main parts:

i. The mobile part (tire, motors and drivers).

ii. Power supply and a regulator circuit.

iii. A computer or the robot's brain.

iv. The wireless system or transceiver.

v. The sensor part.

vi. The monitoring part.

The block diagram of the whole system is shown in Fig. 2, while Fig. 3 (a) and Fig. 3 (b) show the design and the completed mobile robot with the attached LRF respectively.

The power is supplied by two DC batteries - 24 DC voltages and $12 \mathrm{DC}$ voltages. It was designed in such a way to keep the computer works longer. The computer part used 24 DC voltage as shown in red line in Fig. 2. The sensor, i.e. the LRF is powered up by the computer's USB output port which is 5 DC voltages. The $12 \mathrm{DC}$ voltages is used by the motors and drivers. At the monitoring / control part, the power supply is taken directly from the 230 AC supplies which have a regulator to power up the monitor and $\mathrm{AV}$ transceiver. In Fig. 2, the red, orange and yellow line indicate the power line while dark blue, light blue and purple indicate the data line.
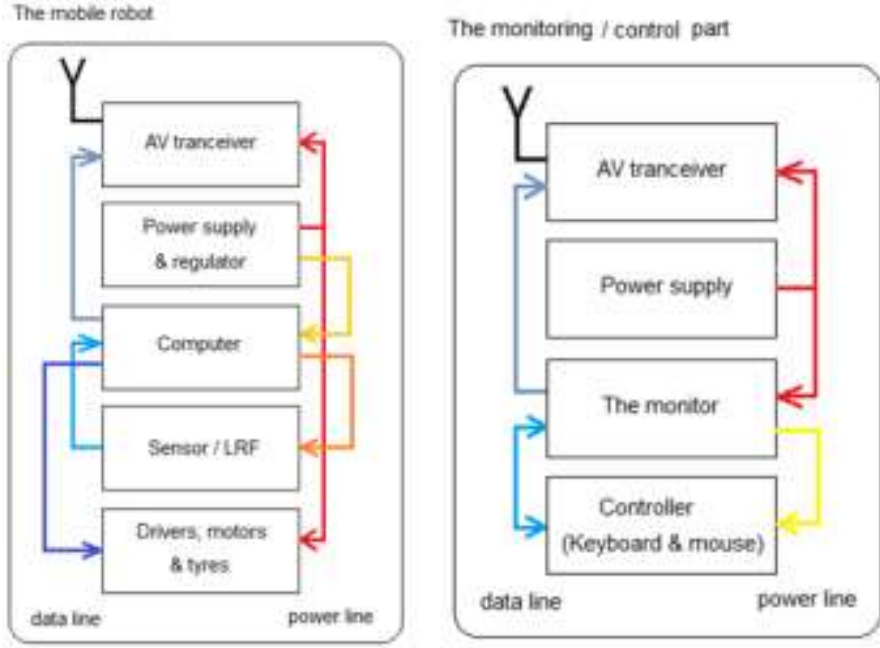

Fig. 2: The mobile robot block diagram and its monitoring system

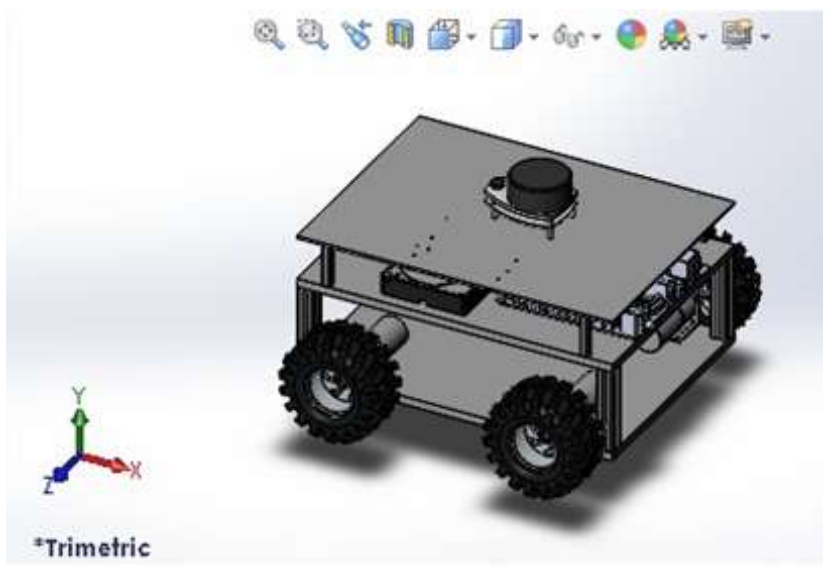

(a)

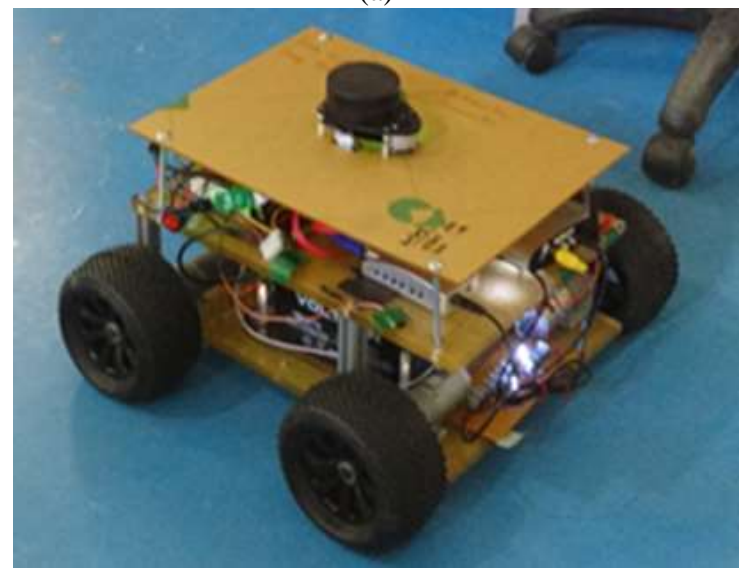

(b)

Fig. 3: (a) The mobile robot design with the RP Lidar (b) The completed mobile robot with the RP Lidar

The mobile part involves four tires, DC motor gears and driver motors. Since the scanning system is wireless, the AV transceiver is used to let the image of the mobile robot's computer to be displayed at the monitor or the monitoring / control part wirelessly in particular distance. Also, this device is able to display the image synchronously between the mobile robot and the monitor / control part. The computer used to attach at the mobile robot is a CPU which is already 
installed with the LRF software development kits (SDK) and other necessary robot software.

As mentioned above, the LRF used in this study is the RP Lidar, developed by Robopeak. It is a low cost LRF and able to scan 360 degrees within $6 \mathrm{~m}$ of range. RP Lidar measures a distance based on a laser triangulation ranging principle and it uses high-speed vision acquisition [15]. Mechanically, it emits modulated infrared laser signal and the laser signal is then reflected by the object to be detected. The returning signal is sampled by a vision acquisition system in the RP Lidar. Then, the DSP embedded in the RP Lidar starts processing the sample data and out with distance and angle value between object and RP Lidar through its communication interface. The RP Lidar is provided with a USB adapter which allows it to communicate with personal computer. In the personal computer, a software development kit (SDK) is ready to save the results of scanning. The scan results will give the user three parameters, which are angles, distances as well as the quality of the results as shown in Table 1. Higher value of quality is better and preferred.

TABLE I

THE RP LIDAR SCAN DATA RESULTS

\begin{tabular}{lll}
\hline \hline \multicolumn{1}{c}{ \#Angule $\left({ }^{\theta_{\text {sts }}}\right)$} & \#Distance $\left({ }^{d}\right)$ & \#Quality \\
\hline \hline 20.7188 & 5430 & 11 \\
\hline 33.875 & 3881 & 10 \\
\hline 37.0938 & 4299.5 & 11
\end{tabular}

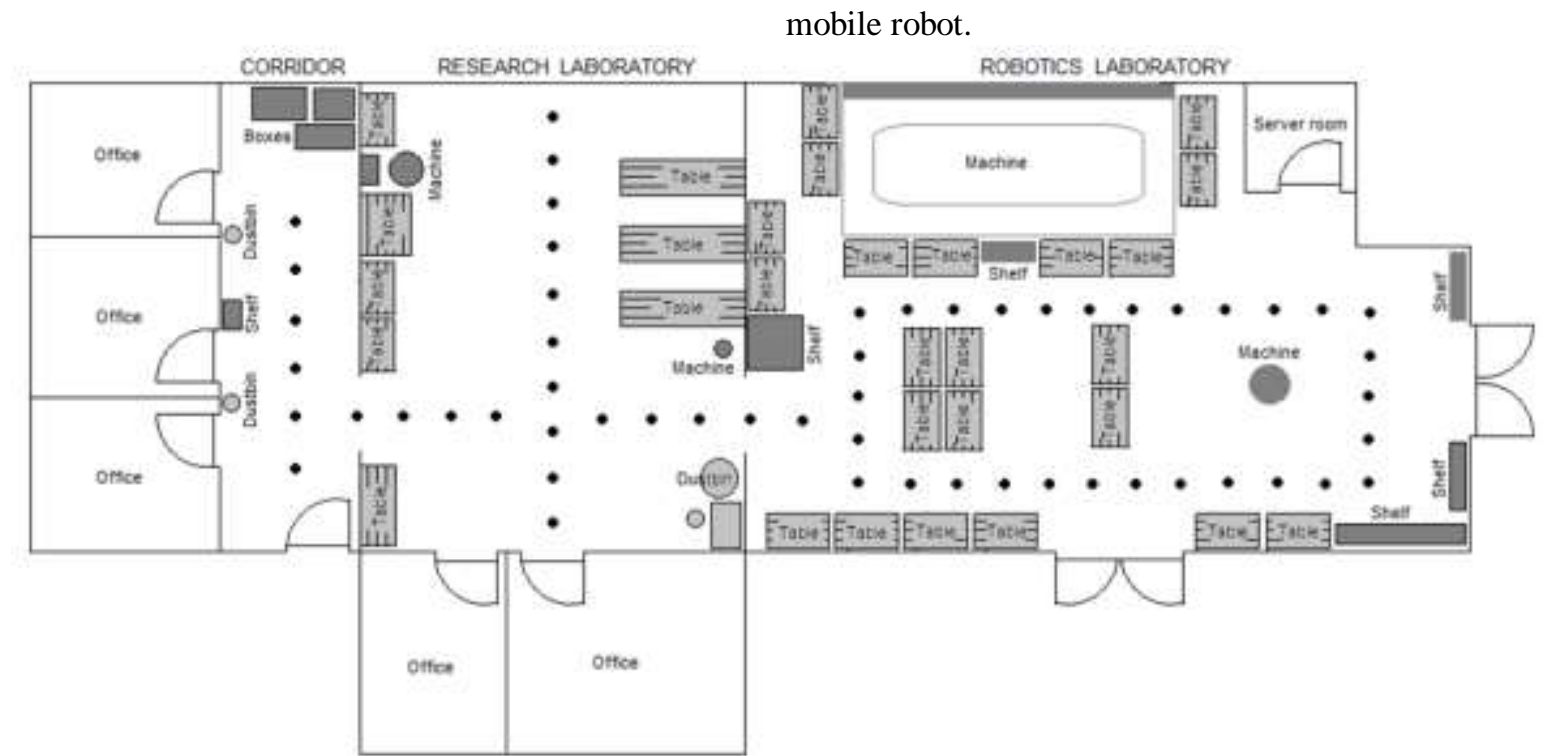

Fig. 4: The environment plan and the mobile robot navigation path

\section{The ScAnNing and Data Collection}

For scanning and data collection, an SDK developed by Robopeak has been used. The SDK main window is shown in Fig. 5. It has a few main buttons such as button to save the scanning data, button to start the scanning as well as button to refresh the SDK. Most of the SDK window will show the

\section{The NAVIGATION}

In this study, the mobile robot is operated in a known environment, hence, the easiest navigation to be adapted is path following. The path following allows the robot to move and navigate by following the path that has been programmed prior to the navigation process. The path can be seen in Fig. 6 which indicated by black points. By the time the mobile robot moving through the black points, it will stop moving to scan the environment for a while. Once the data of the scanning are completely saved in the computer, the mobile robot will continue to move until it reaches the end point.

The navigation and scanning are programmed in the same file. Below is the flow of the mobile robot in order to do the navigation and scanning;

i. The mobile robot takes place at the initial point.

ii. Recognise the point and stop moving.

iii. Do a 360 degree scanning and save the data and repeat 30 times.

iv. Then, move $1 \mathrm{~m}$ forward or move right or left depending on area.

Repeat procedure ii and iv till the last point.

\section{THE ENVIRONMENT}

Three environments have been used to verify the functionality of the mobile robot in scanning and mapping its environment. They are a corridor, a research laboratory and a robotic laboratory. The laboratories are cluttered with equipment and tables. Fig. 4 shows the environments and the respective navigation paths that should be followed by the mobile robot. 


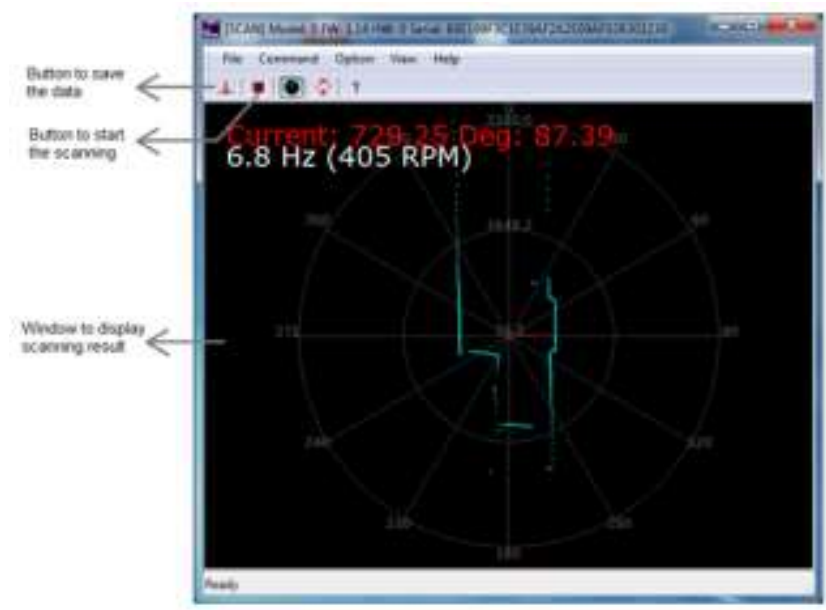

Fig. 5: The SDK for data collection

\section{THE MAPPING}

There are two types of map to be plotted, a local map and a global map. A local map is defined as a current map that is scanned by the LRF of the mobile robot at the particular time. A global map is defined as the complete map of the whole environment. In order to illustrate a global map for an environment, the entire local map in the environment has to map together in one figure as referred to the point in the environment.

Based on the data results, for each angle and distance, the $x$-position and the $y$-position can be calculated using equations below;

i. Convert the degree to radian

$$
\theta_{\text {rad }}=\theta_{\text {deg }} \times \frac{\pi}{180}
$$

ii. Find the $x$ and $y$

$$
\begin{gathered}
x=d \times \sin \left(\theta_{\text {rad }}\right) \\
y=d \times \cos \left(\theta_{\text {rad }}\right)
\end{gathered}
$$

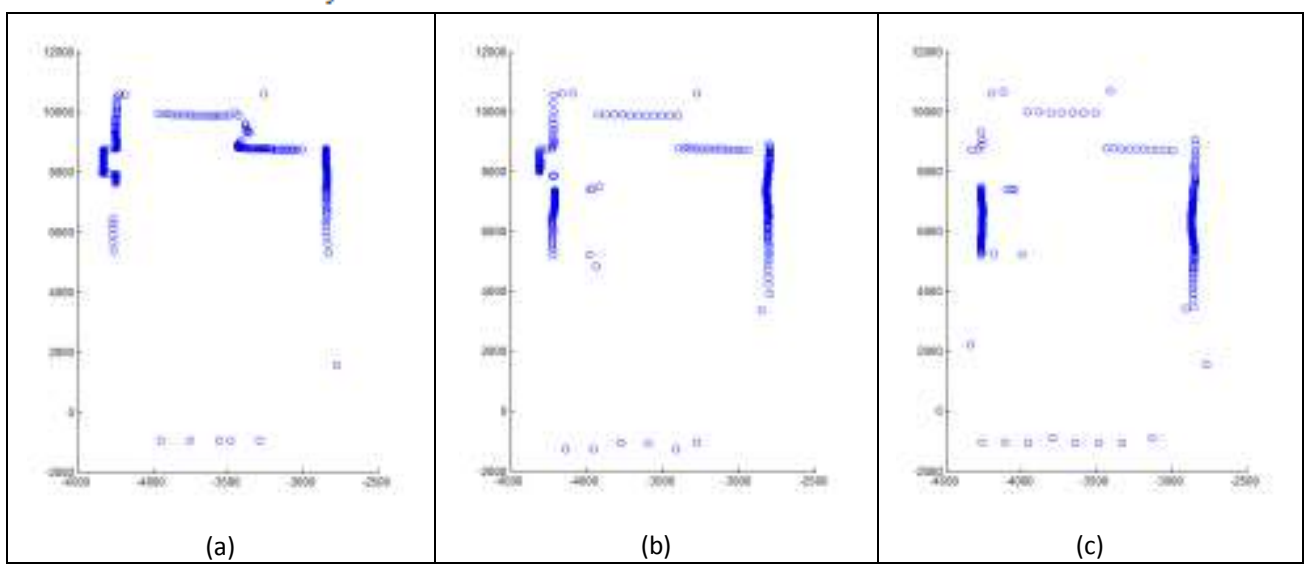

where $\theta_{\text {rad }}$ is an angle of the location in radian and $\theta_{\text {deg }}$ is the angle in degree. $x$ is the position in $x$-axis and $y$ is the $y$ axis that shows the location of the mobile robot. The distance value is a distance from the laser to the obstacle and it defines in millimeters. After obtaining the $\mathrm{x}-\mathrm{y}$ positions in each scanning point, the area of the point is plotted by using scattered plot function in MATLAB.

Each scan point in the map can be defined as a local map. Once all the local maps in the particular environment are obtained, a global map can be developed. The development of a global map is performed by combining the local maps which are referred to its positions.

\section{RESULTS AND DISCUSSIONS}

Once the scanning and data collection work completed, the data is mapped using MATLAB software. MATLAB is used because it provides a platform to do data processing and analysis. In this paper, only the local map of the corridor is shown as in Fig. 6 (a) to (f). There are six local maps for the corridor because it only has six points to navigate and scan.

Fig. 7 (a) to (c) shows the global map for the corridor, research and robotic laboratory as well as its real environment. Based on observation, the plotted maps are similar to the real environment especially for Fig. 7 (a) and (b). However, there are certain angle and small area that cannot be reached by the LRF. This may cause uneven and rough surfaces towards the map. Also, it caused by the limited scanning points. But, these problem can be corrected by applying pre-processing method such as such as filters (raw [16], smooth [17, 18], median [19, 20]) as well as other methods such as histograms [21]. 


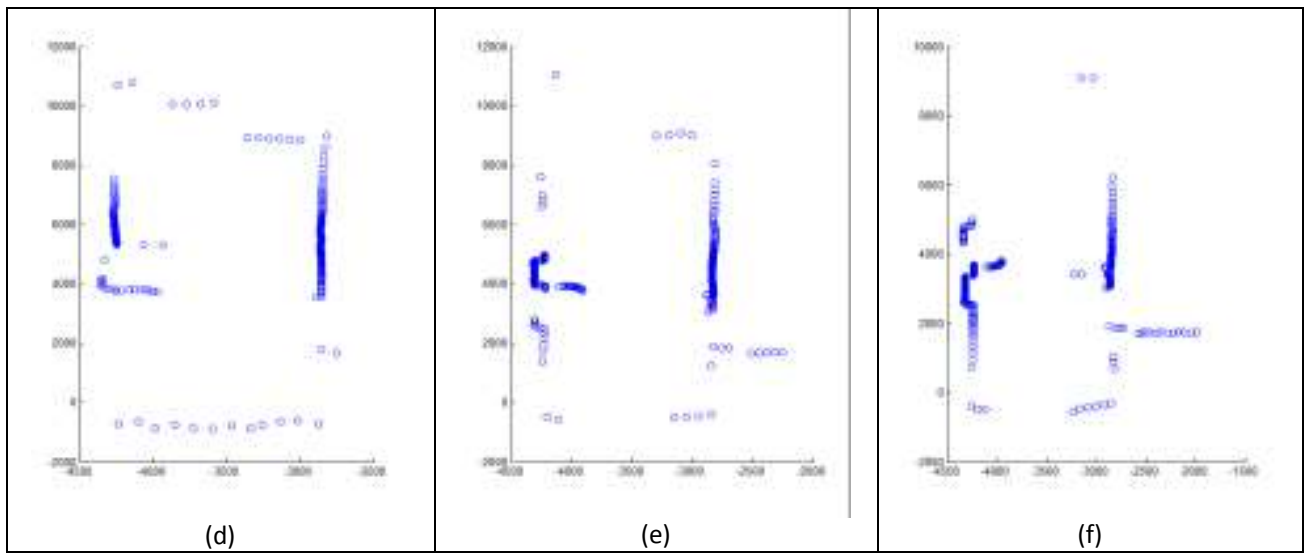

Fig. 6: The corridor local map (a) the local map from point 1 (b) the local map from point 2 (c) the local map from point 3 (d) the local map from point 4 (e) the local map from point 5 (f) the local map from point 6.

be as good as the expensive LRF in the market. Also, the

\section{CONCLUSION}

A mobile robot with a LRF for environmental scanning has successfully developed. The performance of the RP Lidar as the scanning sensor is excellent and being a low cost, it can

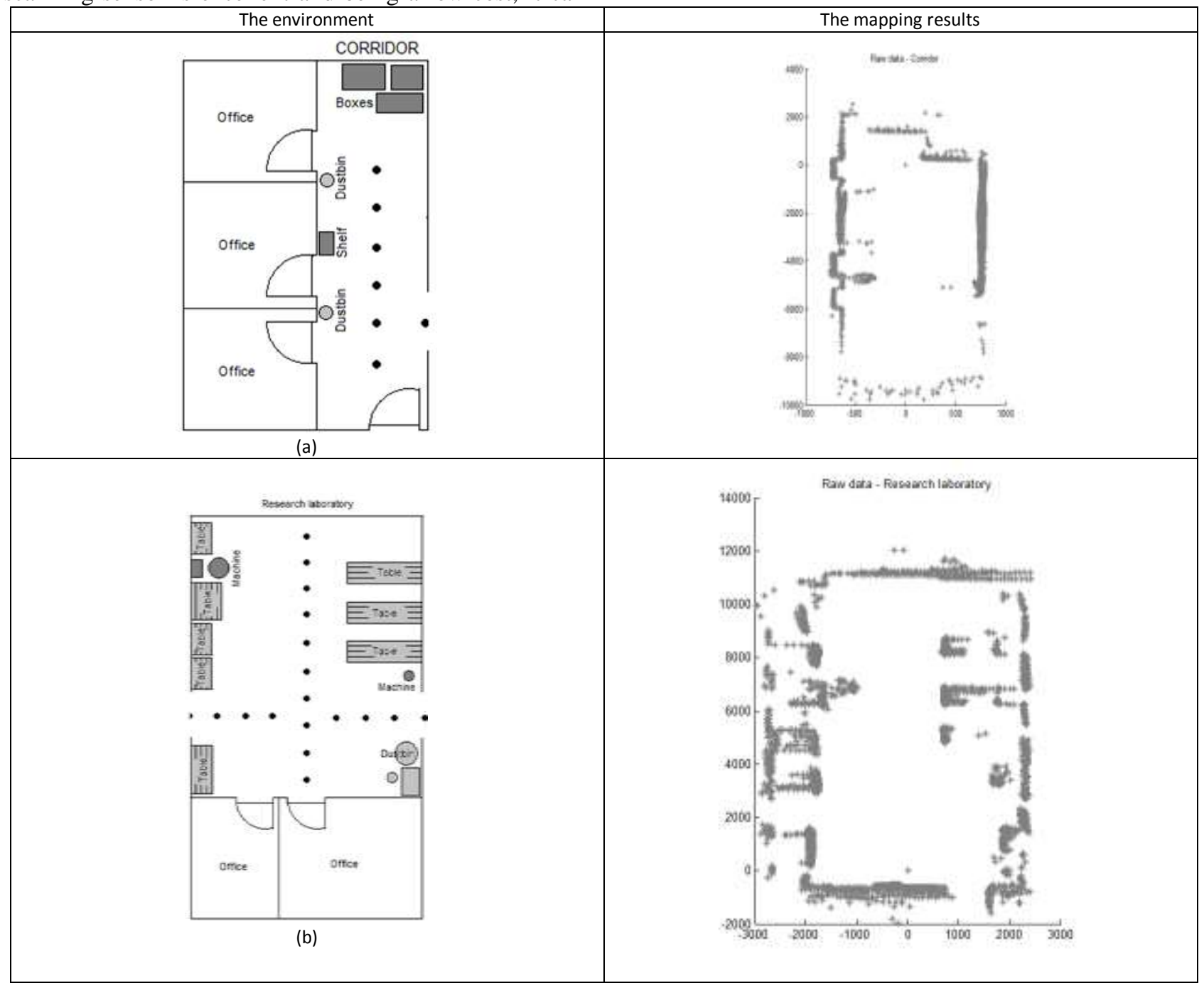
resulting data are easy to handle for future data processing and implementations, such as, for local and global map development as well as scan matching. 


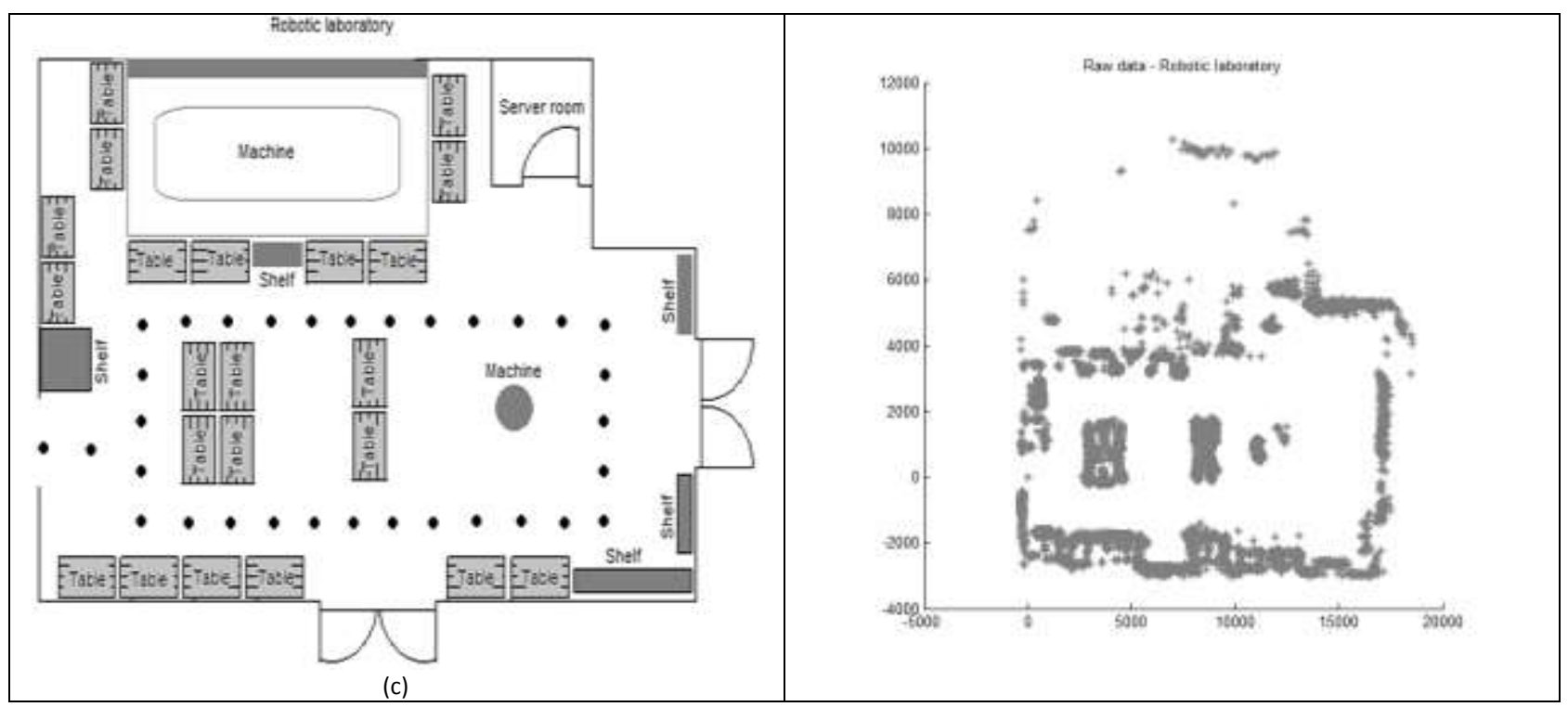

Fig. 7: The global map and its floor plan (a) the corridor (b) the research laboratory, and (c) the robotic laboratory

\section{REFERENCES}

[1] Y. Liu and Y. Sun, "Mobile robot instant indoor map building and localization using 2D laser scanning data", International conference on System Science and Engineering (ICSSE) 2012, June 30 2012-July 2 2012. Dalian, Liaoning. Pp $339-344$. http://dx.doi.org/10.1109/icsse.2012.6257203

[2] Y. S. Chou and J. S. Liu (2013) A robotic indoor 3D mapping system using a 2D laser range finder mounted on a rotating four-bar linkage of a mobile platform, International Journal of Advanced Robotic Systems, Vol. 10.

http://dx.doi.org/10.5772/54655

[3] S. Thrun, W. Burgard and D. Fox, "A real-time algorithm for mobile robot mapping with application to multi-robot and 3D mapping," Proc. IEEE Int. Conf. Robotics and Automation, 2000, pp. 321-328. http://dx.doi.org/10.1109/robot.2000.844077

[4] S. A. Abdul Shukor, Young, K. and E. J. Rushforth, "3D modelling of indoor surfaces with occlusion and clutter", In: IEEE (Institute of Electrical and Electronics Engineers), International Conference on Mechatronics. Istanbul, Turkey 13-15 April 2011. IEEE, pp. 282-287.

[5] C. Ye and J. Borenstein, "Characterization of a 2-D laser scanner for mobile robot obstacle negotiation," Proc. IEEE Int. Conf. Robotics and Automation, 2002, pp. 2512-2518.

[6] R. Philippsen, "Motion planning and obstacle avoidance for mobile robots in highly cluttered dynamic environments", $\mathrm{PhD}$ dissertation, Swiss Federal Institute of Technology, 2004.

[7] J. L. Martínez, J. González, J. Morales, A. Mandow and A. J. GarcíaCerezo (Jan, 2006) Mobile robot motion estimation by 2D scan matching with genetic and iterative closest point algorithms. Journal of Field Robotics. Volume 23, Issue 1, pages 21-34. http://dx.doi.org/10.1002/rob.20104

[8] V. D. Hoang; D. C. Hernandez, M. H. Le, K. H. Jo, "3D motion estimation based on pitch and azimuth from respective camera and laser rangefinder sensing" IEEE/RSJ International Conference on intelligent robots and systems (IROS), 2013. pp 735-740.

[9] F. Lu and E. Milios (1997) Robot pose estimation in unknown environments by matching 2D range Scans. Journal of Intelligent and Robotic Systems, March 1997, Volume 18, Issue 3, pp 249-275. http://dx.doi.org/10.1023/A:1007957421070

[10] X. Wang, Y. Jia, N. Xi, J. Zhen and F. Xu "Mobile robot pose estimation using laser scan matching based on fourier transform. Proc. of IEEE International Conference on Robotics and Biomimetics (ROBIO), Shenzhen, China, December 2013. pp 474-479.

[11] A. Mendes, L. C. Bento and U. Nunes, "Multi-target detection and tracking with a laser scanner", IEEE symposium on intelligent vehicle, University of Palma, Italy, June 14-17 2004.

[12] P. Kondaxakis, S. Kasderidis, P. Trahanias, "A multi-target tracking technique for mobile robots using a laser range scanner" IEEE/RSJ
International Conference on Intelligent Robots and Systems. Acropolis Convention Center Nice, France, Sept, 22-26, 2008.

[13] B. A. Schultz, and W. Adams, "Mobile robot exploration and map-building with continuous localization", IEEE international conference on robotics and automation, 1998. Vol. 4, 16-20 May 1998. Leuven. 3715 - 3720.

[14] Cytron. (29 April 2015). Laser range finder. [online]. Available: http://www.cytron.com.my/c-85-sensor/c-288-laser-range-finder

[15] RP Lidar: Low cost 360 degree 2D laser scanner (Lidar) system Introduction and Datasheet. Robopeak Team. 2014.

[16] F. Duchon, M. Dekan, L. Jurisica and A. Vitco, "Some Applications of Laser Range Finder in Mobile Robotics", CEAI, Vol 14, No 2, 2012, pp 50-57.

[17] The MathWork Inc. (5 May 2015) Filtering and smoothing data, [online] Available:http://www.mathworks.com/help/curvefit/smoothing-data.html.

[18] Y. S. Hwang, D. J. Lee and J. Y. Lee. "3D map building for a moving based on mobile robot", SICE Annual Conference 2014, September 9-12, 2014, Hokkaido University, Sapporo, Japan. 474 - 479

[19] T. Chen and H. R. Wu, "Application of partition-based median type filters for suppressing noise in images," IEEE Trans. Image Processing, vol. 10, no. 6, pp. 829-836, 2001. http://dx.doi.org/10.1109/83.923279

[20] H. Eng and K. Ma, "Noise adaptive soft-switching Median Filter," IEEE Trans. Image Processing, vol. 10, no. 2, pp. 242-251, 2001. http://dx.doi.org/10.1109/83.902289

[21] S.A. Abdul Shukor and E.J. Rushforth, "Adapting histogram for Automatic Noise Data Removal in Building Interior Point Cloud Data", AIP 2014 Conference Proceedings 1660, 070074 (2015); doi: $10.1063 / 1.4915792$.

http://dx.doi.org/10.1063/1.4915792 